\title{
Hemoglobin A1c Risk Score for the Prediction of Coronary Artery Disease in Subjects with Angiographically Diagnosed Coronary Atherosclerosis
}

\author{
En-Zhi Jia ${ }^{a}$ Feng-Hui An ${ }^{b}$ Zhao-Hong Chen ${ }^{b}$ Li-Hua Li $^{b}$ Hong-Wei Mao ${ }^{b}$

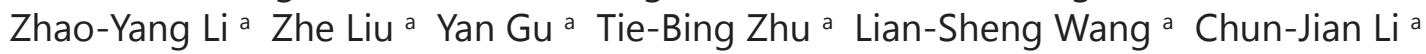 \\ Wen-Zhu Maa Zhi-Jian Yanga \\ aDepartment of Cardiovascular Medicine, the First Affiliated Hospital of Nanjing Medical University, \\ Nanjing, China; bepartment of Cardiovascular Medicine, the Friendship Hospital of Ili Kazakh \\ Autonomous Prefecture, Ili, Xinjiang Uygur Autonomous Regions, China
}

\section{Key Words}

Hemoglobin A1c • Coronary heart disease $\cdot$ Receiver-operating characteristic curve analysis • Area under the curve

\begin{abstract}
Objective: To develop a risk score by incorporating Hemoglobin $\mathrm{A} 1 \mathrm{c}(\mathrm{HbA} 1 \mathrm{c})$ with traditional risk factors for the prediction of coronary artery disease (CAD) in Chinese subjects. Methods: A total of 196 consecutive subjects ( 131 males and 65 females) aged 38-89 years who underwent coronary angiography were enrolled in this study. HbAlc risk score sheets for the prediction of $C A D$ were developed using age, gender and $\mathrm{HbAlc}$. A receiver-operating characteristic curve analysis was used to determine the optimum cut-off levels of the HbAlc risk score for predicting CAD. Results: In the ROC curve analysis, the optimal cut-off value of the HbAlc score for predicting CAD was 5.1, with a sensitivity of $72.0 \%$ and a specificity of $75.5 \%$ (area under the curve $0.781,95 \%$ confidence interval 0.709 to $0.854, p=0.000$ ). Conclusions: The $\mathrm{HbA1c}$ score system is a simple and feasible method that can be used for the prediction of CAD. Large-scale studies are needed to further substantiate these results.
\end{abstract}

E.-Z. Jia and F.-H. An contributed equally to this paper. 
Jia et al.: HbA1c and CAD

\section{Introduction}

Glycated hemoglobin or glycosylated hemoglobin (HbA1c) is a form of hemoglobin that is measured primarily to identify the average plasma glucose concentration over prolonged periods of time. It is formed in a non-enzymatic glycation pathway by hemoglobin's exposure to plasma glucose. As the average amount of plasma glucose increases, the fraction of glycated hemoglobin increases in a predictable way. This serves as a marker for average blood glucose levels over the previous months prior to the measurement [1]. Recently, it has been reported that high HbA1c levels are associated with an increased risk of all-cause mortality and death from cardiovascular disease, coronary heart disease, and cerebral infarction in the general East Asian population, similar to Western populations [2]. Further, subjects with a HbA1c $\geq 6.5 \%$ have a more unfavorable cardiovascular and metabolic risk profile than those with $\mathrm{HbA} 1 \mathrm{c}<6.5 \%$, especially in the oral glucose tolerance test (OGTT)negative Chinese population [3]. A parallel nested case-control study suggested that HbA1c is associated with coronary heart disease risk among apparently healthy, non-diabetic women and men and may be an important early clinical marker of disease risk [4]. HbA1c is significantly associated with the complexity of coronary lesions, an association that is even observed in non-diabetic adults [5]. However, another study concluded that glycated albumin, not $\mathrm{HbA1c}$, was independently associated with significant coronary heart disease in type 2 diabetic patients [6]. The predictive value of HbA1c for coronary artery disease (CAD) has not been elucidated. Therefore, we aimed to develop a risk score incorporating HbA1c with traditional risk factors for the prediction of CAD in Chinese subjects. We hypothesized that a high HbA1c score would be associated with CAD based on the angiography.

\section{Materials and Methods}

Study subjects

From 2010 to 2013, 196 consecutive subjects (131 males and 65 females) age 38-89 years who underwent coronary angiography for suspected or known coronary atherosclerosis at the First Affiliated Hospital of Nanjing Medical University and the Friendship Hospital of Ili Kazakh Autonomous Prefecture in China were enrolled in this study. The exclusion criteria included: subjects with spastic angina pectoris, infectious processes within 2 weeks, heart failure, adrenal dysfunction, and thyroid dysfunction. This study was approved by the ethics committee of the First Affiliated Hospital of Nanjing Medical University and the Friendship Hospital of Ili Kazakh Autonomous Prefecture in China. All subjects gave written informed consent.

\section{Coronary Angiography}

Coronary arteries were cannulated by either the Judkins technique [7] or through a radial approach with $6 \mathrm{~F}$ catheters and recorded at a rate of 30 frames/s. The presence of coronary artery stenosis was evaluated after the direct intracoronary injection of isosorbide dinitrate (ISDN; $2.5 \mathrm{mg} / 5 \mathrm{ml}$ solution over $20 \mathrm{~s}$ ). One minute after the injection of ISDN through the Judkins catheter, coronary angiography was performed from several projections after the injection of ISDN through the Judkins catheter for one minute. Significant CAD was diagnosed if there was a $50 \%$ stenosis in the diameter of at least one major epicardial coronary artery or main tributary $[8,9]$.

\section{Cigarette smoking}

Cigarette smoking was assessed by a standardized questionnaire. Subjects' smoking status was classified as either "never smoking" or "smoking" (the latter group included both former and current smokers).

\section{Blood pressure measurement}

Blood pressure was measured in the right arm with the participant seated and the arm exposed. Three readings were recorded for each individual, and the mean value of the systolic (SBP, $\mathrm{mmHg}$ ) and diastolic blood pressure (DBP, $\mathrm{mmHg}$ ) was recorded. 
Table 1. Subject characteristics and angiographic CAD. HDL-c, fasting high-density lipoprotein cholesterol; LDL-c, fasting low-density lipoprotein cholesterol; SBP, systolic blood pressure; DBP, diastolic blood pressure

\begin{tabular}{lcccc}
\hline Characteristic & $\begin{array}{c}\text { Non-CAD } \\
(\mathrm{n}=53)\end{array}$ & $\begin{array}{c}\text { CAD } \\
(\mathrm{n}=143)\end{array}$ & F or chi-square test & $P$ \\
\hline Age (years) & $57.74 \pm 10.95$ & $62.97 \pm 10.39$ & -3.085 & 0.002 \\
Sex (male/female) & $26 / 27$ & $105 / 38$ & 10.361 & 0.001 \\
Smoking status (yes/no) & $16 / 37$ & $63 / 80$ & 3.091 & 0.079 \\
Total cholesterol (mmol/L) & $4.55 \pm 0.90$ & $4.51 \pm 1.28$ & 0.802 & 0.042 \\
Triglycerides (mmol/L) & $1.38(1.12 \sim 2.06)$ & $1.59(1.17 \sim 2.20)$ & -0.996 & 0.319 \\
Glucose (mmol/L) & $5.09(4.80 \sim 5.70)$ & $5.63(4.93 \sim 6.73)$ & -2.805 & 0.005 \\
HDL-C (mmol/L) & $1.25(1.07 \sim 1.54)$ & $1.24(1.03 \sim 1.58)$ & -0.366 & 0.714 \\
LDL-C (mmol/L) & $2.73 \pm 0.63$ & $2.74 \pm 1.05$ & -0.120 & 0.905 \\
SBP(mmHg) & $130(120 \sim 140)$ & $130(120 \sim 140)$ & -1.421 & 0.155 \\
DBP(mmHg) & $80(72 \sim 90)$ & $80(70 \sim 85)$ & -1.030 & 0.303 \\
HbA1c & $5.70(5.40 \sim 6.20)$ & $6.20(5.70 \sim 7.25)$ & -3.707 & 0.000 \\
HbA1c score & $4.61(4.25 \sim 5.09)$ & $5.48(4.89 \sim 6.30)$ & -6.043 & 0.000 \\
\hline
\end{tabular}

\section{Laboratory measurements}

Total cholesterol (TCH, mmol/L), triglyceride (TG, mmol/L), fasting blood glucose (FBG, mmol/L), fasting high-density lipoprotein cholesterol (HDL-c, mmol/L) and fasting low-density lipoprotein cholesterol (LDL-c, mmol/L) were determined by enzymatic procedures on an automated autoanalyzer (AU 2700 Olympus, 1st Chemical Ltd, Japan). HbA1c was measured by a high-pressure lipid chromatographic assay (VARIANT, BIO-RAD Lab., Hercules, CA, USA)

\section{Statistical analysis}

Data were statistically analyzed using Statistics Package for Social Sciences (ver. 16.0; SPSS Incorporated, Chicago, IL, USA). Subjects were classified into 2 groups according to CAD status. Data for age, $\mathrm{TCH}$, and LDL-c were normally distributed parameters and presented as the mean $\pm \mathrm{SD}$, and comparisons were analyzed by the independent-samples T test. Skewed data, including the TG, FBG, fasting HDL-C, SBP, DBP, HbA1c, and HbA1c score level, were expressed as median and quartile ranges, and comparisons were analyzed by the Mann-Whitney U test. Categorical variables of gender and smoking status were compared between the groups of patients by a chi-squared analysis. Odds ratios $(O R)$ for the presence of obstructive CAD were determined by logistic regression and are presented with $95 \%$ CI. HbA1c risk score sheets for the prediction of CAD were developed using age, gender and HbA1c. The sheet adapted the results of logistic regressions by use of a system that assigned points for each risk factor based on the value for the corresponding $\beta$-coefficient of the regression analyses [10]. According to the univariate logistic regression results, the equation for the HbA1c risk score was as follows: HbA1c score $=0.047 \times$ age $-1.054 \times \operatorname{sex}(\mathrm{male}=1$. female $=2)+0.604 \times \mathrm{HbA1}$. $R O C$ curve analysis was used to determine the optimum cut-off levels of age, $\mathrm{HbA1}$, and the HbA1c risk score that predicted CAD. Differences were considered to be significant if the null hypothesis could be rejected with $>95 \%$ confidence. All $p$-values are two-tailed.

\section{Results}

Baseline Characteristics of the subjects in the study

A total of 196 subjects (131 males and 65 females) with a mean age of 61.55 years were enrolled in the study, there were 30 subjects with acute myocardial infarction, 100 subjects with unstable angina pectoris, and 66 subjects with chest pain. The demographic and clinical characteristics of the subjects are presented in Table 1 . There were significant differences between CAD and non-CAD subjects with respect to age $(p=0.002)$, sex $(p=0.001)$, total cholesterol $(p=0.042)$, fasting glucose $(p=0.005), \mathrm{HbA} 1 \mathrm{c}(p=0.000)$, and $\mathrm{HbA} 1 \mathrm{c}$ score $(p=0.000)$, However, there was no statistically difference in triglyceride $(p=0.319)$, HDL-c $(p=0.714)$ and LDL-c $(p=0.905)$ levels, smoking status ( $p=0.079)$, SBP $(p=0.155)$, and $\operatorname{DBP}(p=0.303)$ between CAD and non-CAD subjects. 


\begin{tabular}{|c|c|c|}
\hline Cellular Physiology & Cell Physiol Biochem 2014;34:672-680 & \\
\hline and Biochemistry & $\begin{array}{l}\text { DOI: } 10.1159 / 000363032 \\
\text { Publisnea onine: August 18, } 2014\end{array}$ & $\begin{array}{l}\text { O } 2014 \text { S. Karger AG, Basel } \\
\text { www.karger.com/cpb }\end{array}$ \\
\hline
\end{tabular}

Table 2. Logistic regression for the presence of obstructive CAD. HDL-c, fasting high-density lipoprotein cholesterol; LDL-c, fasting low-density lipoprotein cholesterol; SBP, systolic blood pressure; DBP, diastolic blood pressure

\begin{tabular}{lcccc}
\hline Characteristic & $\beta$-coefficient & $O R$ & $95 \% C I$ & $P$ \\
\hline Age (years) & 0.047 & 1.048 & $1.016,1.080$ & 0.003 \\
Sex (male=1.female=2) & -1.054 & 0.349 & $0.181,0.670$ & 0.002 \\
Smoking status (yes=1.no=0) & 0.599 & 1.821 & $0.929,3.569$ & 0.081 \\
Total cholesterol (mmol/L) & -0.030 & 0.970 & $0.739,1.274$ & 0.829 \\
Triglycerides (mmol/L) & 0.228 & 1.256 & $0.869,1.814$ & 0.225 \\
Glucose (mmol/L) & 0.272 & 1.312 & $1.040,1.656$ & 0.022 \\
HDL-C (mmol/L) & 0.038 & 1.038 & $0.571,1.890$ & 0.902 \\
LDL-C (mmol/L) & 0.017 & 1.017 & $0.723,1.431$ & 0.923 \\
SBP(mmHg) & 0.013 & 1.013 & $0.994,1.032$ & 0.185 \\
DBP(mmHg) & -0.019 & 0.981 & $0.949,1.014$ & 0.252 \\
HbA1c & 0.604 & 1.829 & $1.269,2.635$ & 0.001 \\
HbA1c score & 1.209 & 3.348 & $2.096,5.348$ & 0.000 \\
\hline
\end{tabular}

Table 3. Receive operating characteristic analysis of age in predicting coronary heart disease. Area under the curve $0.636,95 \%$ confidence interval 0.547 to $0.725, p=0.045$

\begin{tabular}{lccccccc}
\hline Cut-off & Sensitivity & Specificity & Youden index & Cut-off & Sensitivity & Specificity & Youden index \\
\hline 37.0 & 1.000 & 0.000 & 0.000 & 61.5 & 0.580 & 0.623 & 0.203 \\
38.5 & 1.000 & 0.019 & 0.019 & 62.5 & 0.531 & 0.660 & 0.191 \\
39.5 & 1.000 & 0.038 & 0.038 & 63.5 & 0.510 & 0.679 & 0.189 \\
40.5 & 0.979 & 0.038 & 0.017 & 64.5 & 0.469 & 0.698 & 0.167 \\
41.5 & 0.979 & 0.057 & 0.036 & 65.5 & 0.448 & 0.755 & 0.203 \\
42.5 & 0.972 & 0.094 & 0.066 & 66.5 & 0.441 & 0.774 & 0.215 \\
43.5 & 0.965 & 0.094 & 0.059 & 67.5 & 0.399 & 0.774 & 0.173 \\
44.5 & 0.965 & 0.113 & 0.078 & 68.5 & 0.336 & 0.792 & 0.128 \\
45.5 & 0.944 & 0.132 & 0.076 & 69.5 & 0.294 & 0.830 & 0.124 \\
46.5 & 0.930 & 0.189 & 0.119 & 70.5 & 0.259 & 0.830 & 0.089 \\
47.5 & 0.923 & 0.208 & 0.131 & 71.5 & 0.224 & 0.868 & 0.092 \\
48.5 & 0.895 & 0.208 & 0.103 & 72.5 & 0.203 & 0.887 & 0.090 \\
49.5 & 0.860 & 0.283 & 0.143 & 73.5 & 0.168 & 0.906 & 0.074 \\
50.5 & 0.846 & 0.321 & 0.167 & 74.5 & 0.133 & 0.906 & 0.039 \\
51.5 & 0.839 & 0.321 & 0.160 & 75.5 & 0.112 & 0.962 & 0.074 \\
52.5 & 0.818 & 0.377 & 0.195 & 76.5 & 0.091 & 0.962 & 0.053 \\
53.5 & 0.797 & 0.377 & 0.174 & 77.5 & 0.063 & 0.962 & 0.025 \\
54.5 & 0.776 & 0.377 & 0.153 & 78.5 & 0.042 & 0.981 & 0.023 \\
55.5 & 0.776 & 0.396 & 0.172 & 79.5 & 0.021 & 0.981 & 0.002 \\
56.5 & 0.741 & 0.491 & 0.232 & 80.5 & 0.021 & 1.000 & 0.021 \\
57.5 & 0.685 & 0.528 & 0.213 & 82.0 & 0.014 & 1.000 & 0.014 \\
58.5 & 0.643 & 0.566 & 0.209 & 86.0 & 0.007 & 1.000 & 0.007 \\
59.5 & 0.629 & 0.604 & 0.233 & 90.0 & 0.000 & 1.000 & 0.000 \\
60.5 & 0.587 & 0.604 & 0.191 & & & & \\
\hline
\end{tabular}

The logistic regression analysis of characteristics between the CAD and non-CAD groups

The results of the logistic regression analysis of the characteristics between the CAD and non-CAD groups are shown in Table 2. A one year increase was associated with a 4.8\% increased risk for CAD (odds ratio [OR], 1.048; 95\% confidence interval [CI], 1.016-1.080, $p=0.003$ ). Compared to the male subjects, the risk for CAD in female subjects decreased $65 \%$ (odds ratio $[O R], 0.349 ; 95 \%$ confidence interval $[C I], 0.181-0.670, p=0.002$ ). Subjects with a higher HbA1c were $82.9 \%$ more likely to suffer from CAD $(O R, 1.829 ; 95 \%$ CI, 1.269-2.635, $p=0.001$ ). In this population, the HbA1c score was a better indicator of CAD risk than age, sex, and HbA1c alone. Subjects with a higher HbA1c score were $235 \%$ more likely to have CAD (OR, 3.348; 95\% CI, 2.096-5.348, $p=0.000)$.

ROC analysis of age for predicting coronary heart disease

The ROC analysis of age for predicting CAD is shown in Table 3 and Figure 1. The optimal cut-off value of age that predicted CAD was 59.5, with a sensitivity of $62.9 \%$ and a specificity 


\begin{tabular}{|c|c|c|}
\hline Cellular Physiology & Cell Physiol Biochem 2014;34:672-680 & \\
\hline and Biochemistry & $\begin{array}{l}\text { DOI: } 10.1159 / 000363032 \\
\text { Publisned onIIne: August 18, } 2014\end{array}$ & $\begin{array}{l}\text { O 2014 S. Karger AG, Basel } \\
\text { www.karger.com/cpb }\end{array}$ \\
\hline
\end{tabular}

Table 4. Receive operating characteristic analysis of HbA1c in predicting coronary heart disease. Area under the curve $0.672,95 \%$ confidence interval 0.592 to $0.752, p=0.041$

\begin{tabular}{lccccccc}
\hline Cut-off & Sensitivity & Specificity & Youden index & Cut-off & Sensitivity & Specificity & Youden index \\
\hline 3.40 & 1.000 & 0.000 & 0.000 & 7.15 & 0.287 & 0.925 & 0.212 \\
4.45 & 0.993 & 0.019 & 0.012 & 7.25 & 0.259 & 0.925 & 0.184 \\
4.60 & 0.986 & 0.019 & 0.005 & 7.30 & 0.259 & 0.943 & 0.202 \\
4.75 & 0.986 & 0.057 & 0.043 & 7.40 & 0.231 & 0.943 & 0.174 \\
4.85 & 0.986 & 0.075 & 0.061 & 7.55 & 0.210 & 0.943 & 0.153 \\
4.95 & 0.979 & 0.075 & 0.054 & 7.65 & 0.210 & 0.962 & 0.172 \\
5.05 & 0.972 & 0.094 & 0.066 & 7.75 & 0.196 & 0.962 & 0.158 \\
5.15 & 0.958 & 0.113 & 0.071 & 7.85 & 0.175 & 0.962 & 0.137 \\
5.25 & 0.944 & 0.151 & 0.095 & 7.95 & 0.168 & 0.962 & 0.130 \\
5.35 & 0.888 & 0.170 & 0.058 & 8.10 & 0.161 & 0.962 & 0.123 \\
5.45 & 0.860 & 0.264 & 0.124 & 8.25 & 0.147 & 0.962 & 0.109 \\
5.55 & 0.811 & 0.321 & 0.132 & 8.35 & 0.126 & 0.962 & 0.088 \\
5.65 & 0.769 & 0.434 & 0.203 & 8.50 & 0.112 & 0.962 & 0.074 \\
5.75 & 0.692 & 0.509 & 0.201 & 8.70 & 0.105 & 0.962 & 0.067 \\
5.85 & 0.650 & 0.528 & 0.178 & 8.85 & 0.091 & 0.962 & 0.053 \\
5.95 & 0.615 & 0.604 & 0.219 & 8.95 & 0.070 & 0.962 & 0.032 \\
6.05 & 0.559 & 0.660 & 0.219 & 9.05 & 0.063 & 0.962 & 0.025 \\
6.15 & 0.510 & 0.736 & 0.246 & 9.15 & 0.056 & 0.962 & 0.018 \\
6.25 & 0.483 & 0.849 & 0.332 & 9.40 & 0.056 & 0.981 & 0.037 \\
6.35 & 0.455 & 0.868 & 0.323 & 9.90 & 0.049 & 0.981 & 0.030 \\
6.45 & 0.434 & 0.887 & 0.321 & 10.25 & 0.042 & 0.981 & 0.023 \\
6.55 & 0.420 & 0.887 & 0.307 & 10.50 & 0.042 & 1.000 & 0.042 \\
6.65 & 0.399 & 0.925 & 0.324 & 10.85 & 0.028 & 1.000 & 0.028 \\
6.75 & 0.371 & 0.925 & 0.296 & 11.60 & 0.021 & 1.000 & 0.021 \\
6.85 & 0.350 & 0.925 & 0.275 & 12.50 & 0.014 & 1.000 & 0.014 \\
6.95 & 0.343 & 0.925 & 0.268 & 19.40 & 0.007 & 1.000 & 0.007 \\
7.05 & 0.329 & 0.925 & 0.254 & 27.0 & 0.000 & 1.000 & 0.000 \\
\hline
\end{tabular}

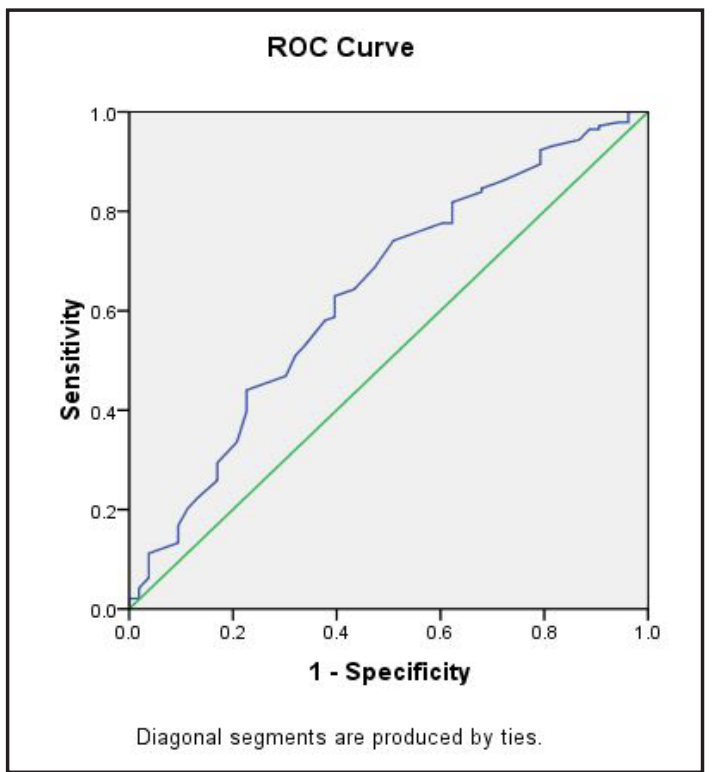

Fig. 1. Receive operating characteristic analysis of age in predicting coronary heart disease.

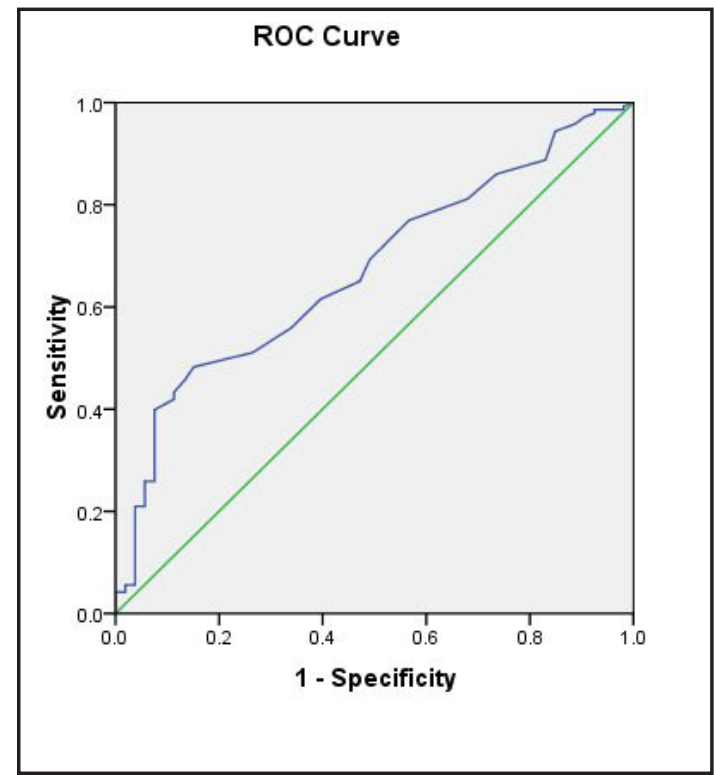

Fig. 2. Receive operating characteristic analysis of $\mathrm{HbA1c}$ in predicting coronary heart disease.

of $60.4 \%$ (area under the curve $0.672,95 \%$ confidence interval 0.592 to $0.752, p=0.041$ ).

ROC analysis of HbA1c for predicting coronary heart disease

The ROC analysis of HbA1c for predicting CAD is shown in Table 4 and Figure 2. The optimal cut-off value of HbA1c that predicted CAD was $6.25 \%$, with a sensitivity of $48.3 \%$ 
Table 5. Receive operating characteristic analysis of HbA1c score in predicting coronary heart disease. Area under the curve $0.781,95 \%$ confidence interval 0.709 to $0.854, p=0.000$

\begin{tabular}{|c|c|c|c|c|c|c|}
\hline Cut-off & Sensitivity & Specificity & Youden index & Cut-off & Sensitivity & Specificity \\
\hline 1.953 & 1.000 & 0.000 & 0.000 & 5.283 & 0.601 & 0.811 \\
\hline 3.074 & 1.000 & 0.019 & 0.019 & 5.296 & 0.594 & 0.811 \\
\hline 3.242 & 1.000 & 0.038 & 0.038 & 5.306 & 0.587 & 0.811 \\
\hline 3.373 & 1.000 & 0.057 & 0.057 & 5.316 & 0.580 & 0.811 \\
\hline 3.480 & 1.000 & 0.075 & 0.075 & 5.323 & 0.580 & 0.811 \\
\hline 3.520 & 1.000 & 0.094 & 0.094 & 5.333 & 0.573 & 0.830 \\
\hline 3.557 & 1.000 & 0.113 & 0.113 & 5.349 & 0.566 & 0.849 \\
\hline 3.651 & 1.000 & 0.132 & 0.132 & 5.356 & 0.559 & 0.849 \\
\hline 3.752 & 0.993 & 0.132 & 0.125 & 5.367 & 0.552 & 0.849 \\
\hline 3.792 & 0.993 & 0.151 & 0.144 & 5.384 & 0.545 & 0.849 \\
\hline 3.829 & 0.993 & 0.170 & 0.163 & 5.393 & 0.538 & 0.849 \\
\hline 3.866 & 0.986 & 0.170 & 0.156 & 5.399 & 0.531 & 0.849 \\
\hline 3.943 & 0.979 & 0.170 & 0.149 & 5.424 & 0.524 & 0.849 \\
\hline 4.051 & 0.972 & 0.170 & 0.142 & 5.460 & 0.524 & 0.868 \\
\hline 4.098 & 0.965 & 0.170 & 0.135 & 5.481 & 0.517 & 0.868 \\
\hline 4.108 & 0.958 & 0.170 & 0.128 & 5.484 & 0.510 & 0.868 \\
\hline 4.124 & 0.958 & 0.189 & 0.147 & 5.487 & 0.497 & 0.868 \\
\hline 4.158 & 0.958 & 0.208 & 0.166 & 5.494 & 0.490 & 0.868 \\
\hline 4.212 & 0.958 & 0.226 & 0.184 & 5.511 & 0.483 & 0.887 \\
\hline 4.249 & 0.958 & 0.245 & 0.203 & 5.548 & 0.476 & 0.887 \\
\hline 4.256 & 0.951 & 0.264 & 0.215 & 5.571 & 0.469 & 0.887 \\
\hline 4.262 & 0.951 & 0.283 & 0.234 & 5.585 & 0.462 & 0.887 \\
\hline 4.269 & 0.944 & 0.283 & 0.227 & 5.599 & 0.455 & 0.887 \\
\hline 4.276 & 0.944 & 0.302 & 0.246 & 5.612 & 0.448 & 0.887 \\
\hline 4.289 & 0.937 & 0.302 & 0.239 & 5.642 & 0.448 & 0.906 \\
\hline 4.313 & 0.937 & 0.321 & 0.258 & 5.665 & 0.441 & 0.906 \\
\hline 4.336 & 0.937 & 0.340 & 0.277 & 5.678 & 0.434 & 0.906 \\
\hline 4.353 & 0.930 & 0.340 & 0.270 & 5.688 & 0.434 & 0.925 \\
\hline 4.376 & 0.923 & 0.340 & 0.263 & 5.722 & 0.427 & 0.925 \\
\hline 4.393 & 0.916 & 0.340 & 0.256 & 5.759 & 0.420 & 0.925 \\
\hline 4.400 & 0.916 & 0.358 & 0.274 & 5.769 & 0.413 & 0.925 \\
\hline 4.407 & 0.909 & 0.358 & 0.267 & 5.799 & 0.406 & 0.925 \\
\hline 4.423 & 0.909 & 0.377 & 0.286 & 5.836 & 0.399 & 0.925 \\
\hline 4.450 & 0.902 & 0.377 & 0.279 & 5.857 & 0.399 & 0.943 \\
\hline 4.474 & 0.902 & 0.396 & 0.298 & 5.870 & 0.392 & 0.943 \\
\hline 4.487 & 0.902 & 0.415 & 0.317 & 5.880 & 0.385 & 0.943 \\
\hline 4.494 & 0.895 & 0.415 & 0.310 & 5.890 & 0.378 & 0.943 \\
\hline 4.507 & 0.895 & 0.434 & 0.329 & 5.900 & 0.371 & 0.943 \\
\hline 4.534 & 0.895 & 0.453 & 0.348 & 5.917 & 0.364 & 0.943 \\
\hline 4.564 & 0.888 & 0.453 & 0.341 & 5.931 & 0.350 & 0.943 \\
\hline 4.581 & 0.888 & 0.472 & 0.360 & 5.940 & 0.343 & 0.943 \\
\hline 4.588 & 0.881 & 0.472 & 0.353 & 5.964 & 0.336 & 0.943 \\
\hline 4.595 & 0.874 & 0.472 & 0.346 & 5.984 & 0.329 & 0.943 \\
\hline 4.605 & 0.874 & 0.491 & 0.365 & 5.988 & 0.322 & 0.943 \\
\hline 4.621 & 0.874 & 0.509 & 0.383 & 5.998 & 0.315 & 0.943 \\
\hline 4.638 & 0.867 & 0.509 & 0.376 & 6.014 & 0.308 & 0.943 \\
\hline 4.645 & 0.860 & 0.509 & 0.369 & 6.021 & 0.301 & 0.943 \\
\hline 4.655 & 0.853 & 0.509 & 0.362 & 6.031 & 0.294 & 0.943 \\
\hline 4.672 & 0.846 & 0.509 & 0.355 & 6.048 & 0.287 & 0.943 \\
\hline 4.682 & 0.839 & 0.528 & 0.367 & 6.054 & 0.280 & 0.943 \\
\hline 4.685 & 0.832 & 0.528 & 0.360 & 6.115 & 0.273 & 0.943 \\
\hline 4.702 & 0.825 & 0.528 & 0.353 & 6.216 & 0.273 & 0.962 \\
\hline 4.729 & 0.818 & 0.528 & 0.346 & 6.256 & 0.266 & 0.962 \\
\hline 4.739 & 0.818 & 0.528 & 0.346 & 6.259 & 0.259 & 0.962 \\
\hline 4.742 & 0.811 & 0.566 & 0.377 & 6.279 & 0.252 & 0.962 \\
\hline 4.749 & 0.804 & 0.566 & 0.370 & 6.299 & 0.238 & 0.962 \\
\hline 4.762 & 0.797 & 0.585 & 0.382 & 6.357 & 0.231 & 0.962 \\
\hline 4.782 & 0.790 & 0.585 & 0.375 & 6.414 & 0.224 & 0.962 \\
\hline 4.799 & 0.790 & 0.604 & 0.394 & 6.441 & 0.217 & 0.962 \\
\hline 4.806 & 0.790 & 0.623 & 0.413 & 6.488 & 0.210 & 0.962 \\
\hline 4.813 & 0.783 & 0.623 & 0.406 & 6.518 & 0.203 & 0.962 \\
\hline 4.826 & 0.776 & 0.623 & 0.399 & 6.538 & 0.196 & 0.962 \\
\hline 4.833 & 0.776 & 0.642 & 0.418 & 6.554 & 0.189 & 0.962 \\
\hline 4.836 & 0.769 & 0.642 & 0.411 & 6.564 & 0.182 & 0.962 \\
\hline 4.846 & 0.762 & 0.642 & 0.404 & 6.578 & 0.175 & 0.962 \\
\hline 4.873 & 0.755 & 0.642 & 0.397 & 6.612 & 0.168 & 0.962 \\
\hline 4.897 & 0.748 & 0.642 & 0.390 & 6.652 & 0.154 & 0.962 \\
\hline 4.907 & 0.741 & 0.642 & 0.383 & 6.679 & 0.147 & 0.962 \\
\hline 4.930 & 0.741 & 0.660 & 0.401 & 6.726 & 0.140 & 0.962 \\
\hline 4.954 & 0.734 & 0.660 & 0.394 & 6.823 & 0.133 & 0.962 \\
\hline 4.967 & 0.734 & 0.679 & 0.413 & 6.900 & 0.126 & 0.962 \\
\hline 4.997 & 0.734 & 0.717 & 0.451 & 6.977 & 0.119 & 0.962 \\
\hline 5.028 & 0.727 & 0.717 & 0.444 & 7.044 & 0.112 & 0.962 \\
\hline 5.034 & 0.720 & 0.717 & 0.437 & 7.071 & 0.105 & 0.962 \\
\hline 5.051 & 0.720 & 0.736 & 0.456 & 7.108 & 0.098 & 0.962 \\
\hline 5.068 & 0.720 & 0.755 & 0.475 & 7.149 & 0.091 & 0.962 \\
\hline 5.071 & 0.713 & 0.755 & 0.468 & 7.202 & 0.084 & 0.962 \\
\hline 5.091 & 0.706 & 0.755 & 0.461 & 7.303 & 0.084 & 0.981 \\
\hline 5.111 & 0.699 & 0.755 & 0.454 & 7.403 & 0.077 & 0.981 \\
\hline 5.115 & 0.699 & 0.774 & 0.473 & 7.464 & 0.070 & 0.981 \\
\hline 5.125 & 0.685 & 0.774 & 0.459 & 7.531 & 0.063 & 0.981 \\
\hline 5.142 & 0.678 & 0.774 & 0.452 & 7.591 & 0.056 & 0.981 \\
\hline 5.169 & 0.671 & 0.774 & 0.445 & 7.709 & 0.049 & 0.981 \\
\hline 5.195 & 0.657 & 0.774 & 0.431 & 7.816 & 0.042 & 0.981 \\
\hline 5.205 & 0.650 & 0.792 & 0.442 & 7.863 & 0.035 & 0.981 \\
\hline 5.219 & 0.643 & 0.792 & 0.435 & 8.034 & 0.028 & 0.981 \\
\hline 5.232 & 0.636 & 0.792 & 0.428 & 8.309 & 0.028 & 1.000 \\
\hline 5.242 & 0.629 & 0.792 & 0.421 & 8.501 & 0.021 & 1.000 \\
\hline 5.256 & 0.622 & 0.792 & 0.414 & 8.611 & 0.014 & 1.000 \\
\hline 5.263 & 0.615 & 0.811 & 0.426 & 13.161 & 0.007 & 1.000 \\
\hline 5.269 & 0.608 & 0.811 & 0.419 & 18.658 & 0.000 & 1.000 \\
\hline
\end{tabular}


Fig. 3. Receive operating characteristic analysis of HbA1c score in predicting coronary heart disease.

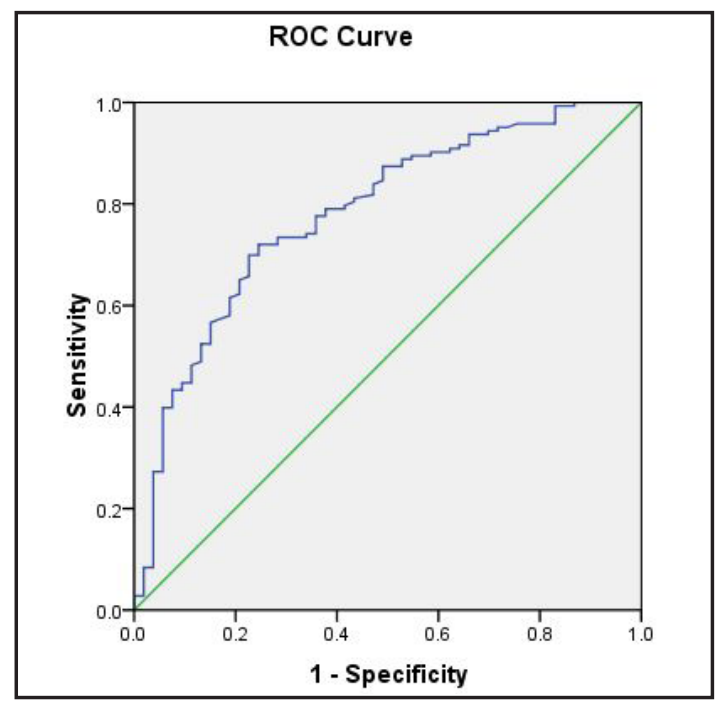

sensitivity and a specificity of $84.9 \%$ (area under the curve $0.672,95 \%$ confidence interval 0.592 to $0.752, p=0.041$ ).

ROC analysis of HbA1c score for predicting coronary heart disease

The ROC analysis of the HbA1c score for predicting CAD is shown in Table 5 and Figure 3. The optimal cut-off value of HbA1c that predicted CAD was 5.1 with a sensitivity of $72.0 \%$ and specificity $75.5 \%$ (area under the curve $0.781,95 \%$ confidence interval 0.709 to 0.854 , $p=0.000$ ).

\section{Discussion}

In the hospital-based diagnostic test of adult Chinese subjects with angiographic evidence of coronary atherosclerosis, the main findings from our study are that the HbA1c score may predict the presence of CAD and that the optimal cut-off value to predict CAD was 5.1 with a sensitivity of $72.0 \%$ and a specificity of $75.5 \%$ (area under the curve $0.781,95 \%$ confidence interval 0.709 to $0.854, p=0.0000$ ).

CAD continues to be a leading cause of morbidity and mortality among adults around the world [11]. Coronary angiography remains the gold standard for defining the presence and severity of CAD in symptomatic patients. However, because slightly more than one third of patients without known disease who undergo elective cardiac catheterization have obstructive $\mathrm{CAD}$, risk stratification in routine clinical practice is needed to increase the diagnostic yield of cardiac catheterization [12]. Though risk algorithms such as Framingham and SCORE are linearly associated with the extent of angiographic disease, a number of common variables such as cholesterol and blood pressure have been shown to be poor indicators of CAD, possibly due to high rates of risk factor modification with statins and antihypertensive therapy. Therefore, a score is necessary to improve diagnostic precision, particularly because the prevalence of non-obstructive CAD on angiography may be greater than $50 \%$ in some populations [9]. The results from a community-dwelling Japanese population suggest that HbA1c levels are an independent risk factor for CAD [13]. Another study indicated that $\mathrm{HbA1c}$ was an independent risk factor for the presence of severe CAD (Beta $=0.374, p<0.001)$. In a ROC curve analysis, the optimal cut-off value of HbA1c that predicted severe CAD was $6.52 \%$ with a sensitivity of $74.4 \%$ and a specificity of $75.1 \%$ (area under the curve $0.781,95 \%$ confidence interval 0.661 to $0.901, p<0.001$ ) [14]. However, the results of this study were limited to subjects who were less than 40 years old. The ideal cut-off value of HbA1c for predicting the occurrence of CAD in non-diabetic subjects was 5.6\% (sensitivity: $60.5 \%$, specificity: $52 \%$ ) [15]. In the present study, the optimal cut-off 
value of $\mathrm{HbA} 1 \mathrm{c}$ that predicted CAD was $6.25 \%$, with a sensitivity of $48.3 \%$ and a specificity of $84.9 \%$ (area under the curve $0.672,95 \%$ confidence interval 0.592 to $0.752, p=0.041$ ). These results agree with those from the study that focused on the relationship between $\mathrm{HbA1c}$ levels and the severity of CAD in patients less than 40 years old.

Easy-to-perform and reliable parameters are needed to predict the presence of CAD and to implement efficient diagnostic and therapeutic modalities. The Framingham risk scoring system has been studied to determine its diagnostic value in detecting CAD. A cut-off level of 7.5 for the Framingham risk scoring system predicted severe CAD with a sensitivity of $68 \%$ and a specificity of 73.6\% (ROC area under curve: $0.776,95 \% C I$ : $0.706-0.845$, PPV: $78.1 \%$, NPV: $62.3 \%, p<0.0001$ [16]. However, the Framingham risk scoring system predicts the presence of CAD by taking into account sex, age, blood lipid, blood pressure, and a history of diabetes and smoking. Due to the high rates of risk factor modification with statins and antihypertensive therapy, the Framingham risk scoring system was not a simple and feasible method for the prediction of CAD. In the present study, the optimal cut-off value of the HbA1c score that consisted of age, sex, and HbA1c predicted CAD with a sensitivity of $72.0 \%$ and a specificity of $75.5 \%$ (area under the curve $0.781,95 \%$ confidence interval 0.709 to 0.854 , $p=0.000$ ).

This study has a number of limitations. For one, the sample size was small and largescale studies are necessary to substantiate our findings. Further, there is a fundamental difference between the Framingham and the present study. The Framingham study was a prospective study that helped to establish the causal relationship between hypertension, high cholesterol, diabetes and CAD. Additionally, the study created risk scores to predict death and coronary heart disease. The association between age, gender, HbA1c and CAD is not original. Though interesting, the practical application of the risk score developed in the present study may be limited and may not substantially change the clinical indication for performing a coronary angiogram.

The present study suggests that the HbA1c score system is a simple and feasible method that can be used to predict CAD. The exact mechanism underlying the association needs further study.

\section{Acknowledgements}

Dr. En-Zhi Jia is a Assistant Fellow at the Collaborative Innovation Center For Cardiovascular Disease Translational Medicine.

Supported by the National Natural Science Foundation of China, No. 30400173, 30971257, and 81170180. A project funded by the Priority Academic Program Development of Jiangsu Higher Education Institutions

\section{References}

1 Larsen ML, Hørder M, Mogensen EF: Effect of long-term monitoring of glycosylated hemoglobin levels in insulin-dependent diabetes mellitus. N Engl J Med. 1990;323:1021-1025.

2 Sakurai M, Saitoh S, Miura K, Nakagawa H, Ohnishi H, Akasaka H, Kadota A, Kita Y, Hayakawa T, Ohkubo T, Okayama A, Okamura T, Ueshima H; for the NIPPON DATA90 Research Group: HbA1c and the Risks for AllCause and Cardiovascular Mortality in the General Japanese Population: NIPPON DATA90. Diabetes Care 2013;36:3759-3765.

- 3 Peng G, Lin M, Zhang K, Chen J, Wang Y, Yang Y, Wang J, Huang H: Hemoglobin A1c Can Identify More Cardiovascular and Metabolic Risk Profile in OGTT-Negative Chinese Population. Int J Med Sci 2013; 10:1028-1034.

4 Pai JK, Cahill LE, Hu FB, Rexrode KM, Manson JE, Rimm EB: Hemoglobin a1c is associated with increased risk of incident coronary heart disease among apparently healthy, nondiabetic men and women. J Am Heart Assoc 2013;2:e000077. 


\begin{tabular}{|c|c|c|}
\hline Cellular Physiolos & Cell Physiol Biochem 2014;34:672-680 & \\
\hline and Biochemistry & $\begin{array}{l}\text { DOI: 10.1159/000363032 } \\
\text { Publisned onlne: August 18, } 2014\end{array}$ & $\begin{array}{l}\text { O } 2014 \text { S. Karger AG, Basel } \\
\text { www.karger.com/cpb }\end{array}$ \\
\hline
\end{tabular}

5 Ikeda N, Iijima R, Hara H, Moroi M, Nakamura M, Sugi K: Glycated hemoglobin is associated with the complexity of coronary artery disease, even in non-diabetic adults. J Atheroscler Thromb 2012;19:10661072.

6 Shen Y, Pu LJ, Lu L, Zhang Q Zhang RY, Shen WF: Glycated albumin is superior to hemoglobin A1c for evaluating the presence and severity of coronary artery disease in type 2 diabetic patients. Cardiology 2012;123:84-90.

7 Judkins MP: A percutaneous transfemoral technique. Radiology1967; 89: 815-821.

8 Shen Y, Pu LJ, Lu L, Zhang Q Zhang RY, Shen WF: Glycated albumin is superior to hemoglobin A1c for evaluating the presence and severity of coronary artery disease in type 2 diabetic patients. Cardiology 2012;123:84-90.

-9 Kotecha D, New G, Flather MD, Eccleston D, Pepper J, Krum H: Five-minute heart rate variability can predict obstructive angiographic coronary disease. Heart 2012;98:395-401.

10 Wilson PW, D'Agostino RB, Levy D, Belanger AM, Silbershatz H, Kannel WB: Prediction of coronary heart disease using risk factor categories. Circulation 1998;97:1837-1847.

11 McGovern PG, Jacobs DR Jr, Shahar E, Arnett DK, Folsom AR, Blackburn H, Luepker RV: Trends in acute coronary heart disease mortality, morbidity, and medical care from 1985 through 1997: the Minnesota heart survey. Circulation 2001;104:19-24.

12 Patel MR, Peterson ED, Dai D, Brennan JM, Redberg RF, Anderson HV, Brindis RG, Douglas PS: Low diagnostic yield of elective coronary angiography. N Engl J Med 2010;362:886-895.

13 Ikeda F, Doi Y, Ninomiya T, Hirakawa Y, Mukai N, Hata J, Shikata K, Yoshida D, Matsumoto T, Kitazono T, Kiyohara Y: Haemoglobin A1c even within non-diabetic level is a predictor of cardiovascular disease in a general Japanese population: the Hisayama Study. Cardiovasc Diabetol 2013;12:164.

14 Ayhan SS, Tosun M, Ozturk S, Alcelik A, Ozlu MF, Erdem A, Erdem K, Erdem FH, Yazici M: Glycated haemoglobin is correlated with the severity of coronary artery disease independently of traditional risk factors in young patients. Endokrynol Pol 2012;63:367-371.

15 Ashraf H, Boroumand MA, Amirzadegan A, Talesh SA, Davoodi G: Hemoglobin A1C in non-diabetic patients: An independent predictor of coronary artery disease and its severity. Diabetes Res Clin Pract 2013;102:225-232.

16 Sayin MR, Cetiner MA, Karabag T, Akpinar I, Sayin E, Kurcer MA, Dogan SM, Aydin M: Framingham risk score and severity of coronary artery disease. Herz Doi 10.1007/s00059-013-3881-3884. 